\title{
Anomalies in Noncommutative Dipole Field Theories
}

\author{
D.H. Correa ${ }^{a *}$, G.S. Lozano ${ }^{b \dagger}$, \\ E.F. Moreno ${ }^{a \dagger}$ and F.A.Schaposnik ${ }^{a \ddagger}$ \\ ${ }^{a}$ Departamento de Física, Universidad Nacional de La Plata \\ C.C. 67, 1900 La Plata, Argentina \\ ${ }^{b}$ Departamento de Física, FCEyN, Universidad de Buenos Aires \\ Pab.1, Ciudad Universitaria, CP 1428, Buenos Aires,Argentina
}

August 23, 2021

\begin{abstract}
We study chiral symmetries of fermionic non commutative dipole theories. By using Fujikawa's approach we obtain explicit expressions of the anomalies for Dirac and chiral fermions in 2 and 4 dimensions.
\end{abstract}

\section{Introduction}

Noncommutative field theories have proven to be a rich nonlocal generalization of ordinary field theories, with appealing connections with string theories (see ref.[1] for a complete list of references). Apart from noncommutative theories in the Moyal plane, basically defined by the coordinate commutator algebra $\left[x_{\mu}, x_{\nu}\right]=i \theta_{\mu \nu}$ (with $\theta_{\mu \nu}$ the constant antisymmetric matrix measuring noncommutativity), other nonlocal field theories, known as "dipole field theories" have recently been proposed [2]-[5]. In fact, a key feature of noncommutative field theories is that they describe the dynamics of dipole-like objects, with electric dipole moment related to their momentum according to [6]-8]

$$
L^{\mu} \sim \theta^{\mu \nu} p_{\mu}
$$

Dipole theories share this property but they are much simpler, their intrinsic dipole vectors $L^{\mu}$ being constants.

Dipole theories were obtained in [2] by studying T-duality of twisted noncommutative gauge theories. They can also be connected with Dp-branes pinned

\footnotetext{
${ }^{*}$ CONICET

${ }^{\dagger}$ Associated with CONICET

$¥$ Associated with CICPBA
} 
by a potential related to the B field [3]. They are also interesting theories per se: they show signs of CP and CPT violations, they allow to define sensible noncommutative $S U(N)$ gauge theories and supersymmetric extensions and they could be used to build up a noncommutative version of the Standard model [5].

In this work we discuss the issue of anomalies in dipole gauge theories. First, we study the $U(1)$ chiral anomaly for a $U(N)$ dipole gauge theory coupled to Dirac fermions both in the fundamental (or anti-fundamental) and adjoint representations. Then, we consider the case in which fermions have a chiral coupling to the gauge field, thus studying gauge anomalies in dipole theories. Our analysis can be summarized as follows. We first consider a dipole theory consisting of Dirac fermions coupled to $U(N)$ gauge fields. We study the conservation of the $U(1)$ chiral current both in $d=2$ and $d=4$ dimensions. Using a path-integral approach, we show that there is a chiral anomaly which, for fermions in the "fundamental" or "antifundamental" representations, could be trivially inferred from the corresponding result in ordinary (commutative) theories by an appropriate renaming of the gauge fields. In contrast, when fermions are considered in the "adjoint" a genuine new and non trivial result is obtained. A distinctive feature should be signaled in comparison with Moyal noncommutative theories: for vanishing dipole length one recovers the trivial result to be expected for fermions in the adjoint in ordinary theories, in contrast with what it happens for Moyal theories due to the IR/UV mixing [9]-15]. We also consider the issue of gauge anomalies in dipole theories with Weyl fermions. Using the Fujikawa approach, we analyze the covariant form of the gauge current anomaly finding in this case that the $U(1)$ and $S U(N)$ contributions have a mixed dependence on the dipole length so that the result cannot be related, even for the fundamental or antifundamental representations, to that in commutative theories by a renaming of the gauge fields.

\section{The chiral anomaly}

Given two functions $\phi(x), \chi(x)$, defined in $d$ dimensional Euclidean space-time, the "dipole star product" is defined as

$$
\phi(x) * \chi(x) \equiv \phi\left(x-\frac{L_{\chi}}{2}\right) \chi\left(x+\frac{L_{\phi}}{2}\right)
$$

where $L_{\phi}\left(L_{\phi}=\left(L_{\phi}^{\mu}\right), \mu=1,2, \ldots, d\right)$ is a constant vector measuring the "dipole length" associated with $\phi$. This noncommutative product is associative provided that

$$
L_{\phi * \chi}=L_{\phi}+L_{\chi} .
$$

Given $N$ functions $\phi^{a}(x)(a=1,2, \ldots, N)$ satisfying

$$
\sum_{a=1}^{N} L_{\phi}^{a}=0
$$


one can use integration over $R^{d}$ to define a trace-like operation

$$
\int d^{d} x \phi^{1} * \phi^{2} * \ldots * \phi^{N-1} * \phi^{N}=\int d^{d} x \phi^{N} * \phi^{1} * \phi^{2} * \ldots * \phi^{N-1} .
$$

Calling $\phi^{\dagger}$ the complex (hermitian) conjugate of $\phi$ one can see that

$$
\left(\phi^{\dagger} * \phi\right)=\left(\phi^{\dagger} * \phi\right)^{\dagger} \Leftrightarrow L_{\phi}=-L_{\phi^{\dagger}} .
$$

For a fermionic field $\psi(x)$, one then has

$$
L_{\bar{\psi}}=-L_{\psi},
$$

while real fields should have zero dipole length. In particular, for gauge fields $A_{\mu}$, one necessarily has

$$
L_{A_{\mu}}=0 \text {. }
$$

This in turn implies that the field strength has the form it takes for ordinary products since, being the dipole length zero, $A_{\mu}(x) * A_{\nu}(x)=A_{\mu}(x) A_{\nu}(x)$.

We shall consider in this section the case of Dirac fermions $\psi$ coupled to $U(N)$ gauge fields, $A_{\mu}(x)=A_{\mu}^{a}(x) t^{a}$ with $t^{a}$ the $U(N)$ generators $\left(a=0,1, \ldots, N^{2}-\right.$ 1), normalized according to

$$
\operatorname{tr}^{\mathrm{g}}\left(t^{a} t^{b}\right)=\frac{1}{2} \delta^{a b}
$$

Under local gauge transformations, $A_{\mu}(x)$ changes as

$$
\begin{aligned}
A_{\mu}(x) \rightarrow A_{\mu}^{g}(x) & =g(x) * A_{\mu}(x) * g^{-1}(x)-\frac{i}{e} \partial_{\mu} g(x) * g^{-1}(x) \\
& =g(x) A_{\mu}(x) g^{-1}(x)-\frac{i}{e} \partial_{\mu} g(x) g^{-1}(x)
\end{aligned}
$$

with $g(x) \in U(N)$. Concerning Dirac fermions, one can define, as for the ordinary Moyal product, gauge transformations in a "fundamental" representation,

$$
\begin{aligned}
& \psi(x) \quad \rightarrow \quad \psi^{g}(x)=g(x) * \psi(x) \\
& \bar{\psi}(x) \quad \rightarrow \quad \bar{\psi}^{g}(x)=\bar{\psi}(x) * g^{-1}(x) .
\end{aligned}
$$

Alternatively, one can consider an "antifundamental" representation with fermions transforming as

$$
\begin{aligned}
& \psi(x) \quad \rightarrow \quad \psi^{g}(x)=\psi(x) * g^{-1}(x) \\
& \bar{\psi}(x) \quad \rightarrow \quad \bar{\psi}^{g}(x)=g(x) * \bar{\psi}(x) .
\end{aligned}
$$

Finally, there is the possibility of fermions in the "adjoint" representation. Under gauge transformations, they change according to

$$
\psi(x) \rightarrow \psi^{g}(x)=g(x) * \psi(x) * g^{-1}(x)
$$


with $\psi=\left(\psi_{i}^{j}\right), i, j=1,2, \ldots, N$. The corresponding covariant derivatives take the form

$$
\begin{aligned}
D_{\mu}^{f}[A] \psi(x) & =i \partial_{\mu} \psi(x)+e A_{\mu}(x) * \psi(x)=i \partial_{\mu} \psi(x)+e A_{\mu}\left(x-\frac{L_{\psi}}{2}\right) \psi(x) \\
D_{\mu}^{\bar{f}}[A] \psi(x) & =i \partial_{\mu} \psi(x)-e \psi(x) * A_{\mu}(x)=i \partial_{\mu} \psi(x)-e \psi(x) A_{\mu}\left(x+\frac{L_{\psi}}{2}\right) \\
D_{\mu}^{a d}[A] \psi(x) & =i \partial_{\mu} \psi(x)+e\left[A_{\mu}(x), \psi(x)\right] \\
& =i \partial_{\mu} \psi(x)+e A_{\mu}\left(x-\frac{L_{\psi}}{2}\right) \psi(x)-e \psi(x) A_{\mu}\left(x+\frac{L_{\psi}}{2}\right) .
\end{aligned}
$$

For each one of the previous cases, one can construct a gauge invariant Dirac action,

$$
\begin{gathered}
S[\bar{\psi}, \psi, A]=\int d^{d} x \bar{\psi}(x) * \gamma^{\mu} D_{\mu}[A] \psi(x) \\
S\left[\bar{\psi}^{g}, \psi^{g}, A^{g}\right]=S[\bar{\psi}, \psi, A]
\end{gathered}
$$

where a trace over the gauge group indices is implicit in the definition of the action.

At the classical level, the action (15) is also invariant under global $U(1)$ chiral rotations. For all three representations the global chiral rotation (with infinitesimal phase $\delta \phi$ ) takes the same form,

$$
\begin{aligned}
& \psi(x) \rightarrow \psi^{\delta \phi}=\exp \left(\gamma_{5} \delta \phi\right) \psi(x) \\
& \bar{\psi}(x) \rightarrow \bar{\psi}^{\delta \phi}=\bar{\psi}(x) \exp \left(\gamma_{5} \delta \phi\right) \\
& S\left[\bar{\psi}^{\delta \phi}, \psi^{\delta \phi}, A\right]=S[\bar{\psi}, \psi, A] .
\end{aligned}
$$

At the quantum level, the fermionic effective action $S_{\text {eff }}[A]$ is defined by

$$
\exp \left(-S_{e f f}[A]\right)=\int D \bar{\psi} D \psi \exp (-S[\bar{\psi}, \psi, A]) \equiv \operatorname{det}(\not D[A])
$$

As in the ordinary case, a path-integral measure for Dirac fermions can be defined so that the resulting effective action is gauge invariant,

$$
S_{\text {eff }}\left[A^{g}\right]=S_{\text {eff }}[A] .
$$

Nevertheless, the chiral invariance of the quantum effective action is not $a$ priori guaranteed, that is, an anomaly could arise.

In order to analyze this issue, we shall follow Fujikawa's approach (see 16][17] for details). In particular, we shall focus on the adjoint representation since as we shall discuss later, the results for the other two cases (fundamental and antifundamental representations) can be trivially derived from the corresponding results in ordinary commutative field theories.

Expanding the fermion field in an appropriate basis $\left\{\varphi_{n}(x)\right\}$ with Grassman coefficients $c_{n}$

$$
\psi(x)=\sum c_{n} \varphi_{n}(x), \quad \bar{\psi}(x)=\sum \bar{c}_{n} \varphi_{n}(x)^{\dagger},
$$


the path-integral measure is formally defined as

$$
D \bar{\psi} D \psi \equiv \Pi d \bar{c}_{n} d c_{n} .
$$

One then promotes the global chiral rotation (17) to a local change of variables in the path-integral. In noncommutative space, there are various possibilities to do this. One can consider the following three

$$
\begin{gathered}
\psi(x) \rightarrow \psi^{\delta \phi}=\exp \left(\gamma_{5} \delta \phi(x)\right) * \psi(x) \\
\bar{\psi}(x) \rightarrow \bar{\psi}^{\delta \phi}=\bar{\psi}(x) * \exp \left(\gamma_{5} \delta \phi(x)\right) \\
\psi(x) \rightarrow \psi^{\delta \phi}=\psi(x) * \exp \left(\gamma_{5} \delta \phi(x)\right) \\
\bar{\psi}(x) \rightarrow \bar{\psi}^{\delta \phi}=\exp \left(\gamma_{5} \delta \phi(x)\right) * \bar{\psi}(x) \\
\psi(x) \rightarrow \psi^{\delta \phi}(x)=\exp \left(\gamma_{5}[\delta \phi(x),]_{*}\right) \psi(x) \\
\bar{\psi}(x) \rightarrow \bar{\psi}^{\delta \phi}(x)=\bar{\psi}(x) \exp \left([, \delta \phi(x)]_{*} \gamma_{5}\right) .
\end{gathered}
$$

To each of these transformations it corresponds the following classically conserved currents

$$
\begin{gathered}
j_{\mu}^{5(1)}(x)=i\left(\gamma_{\mu} \gamma_{5}\right)^{\alpha \beta} \operatorname{tr}^{\mathrm{g}}\left(\psi^{\beta}(x) * \bar{\psi}^{\alpha}(x)\right) \\
j_{\mu}^{5(2)}(x)=i\left(\gamma_{\mu} \gamma_{5}\right)^{\alpha \beta} \operatorname{tr}^{\mathrm{g}}\left(\bar{\psi}^{\alpha}(x) * \psi^{\beta}(x)\right) \\
j_{\mu}^{5(3)}(x)=i\left(\gamma_{\mu} \gamma_{5}\right)^{\alpha \beta} \operatorname{tr}^{\mathrm{g}}\left(\psi^{\beta}(x) * \bar{\psi}^{\alpha}(x)+\bar{\psi}^{\alpha}(x) * \psi^{\beta}(x)\right)
\end{gathered}
$$

respectively. Notice that, in view of the definition of the dipole product,

$$
j_{\mu}^{5(1)}(x)=-j_{\mu}^{5(2)}(x+L)
$$

and then in the commutative $(L \rightarrow 0)$ limit both currents coincide. Concerning $j_{\mu}^{5(3)}(x)$, it is the current which naturally couples in the adjoint to an axial $U(1)$ vector field and it vanishes in the $L \rightarrow 0$ limit.

These change of variables could in principle give rise to a non-trivial Jacobian $J$ in the path-integral measure,

$$
D \bar{\psi}^{\delta \phi} D \psi^{\delta \phi}=J^{2} D \bar{\psi} D \psi
$$

Using that $\delta Z / \delta \phi(x)=0$, one obtains the anomaly equation

$$
\left\langle\partial_{\mu} j_{\mu}^{5}\right\rangle=\mathcal{A}(x)
$$


Let us concentrate for definiteness in the case of current $j_{\mu}^{5(3)}(x)$. The anomaly $\mathcal{A}$ appearing in the r.h.s. of 27 , is related to the Fujikawa Jacobian through the formula

$$
\mathcal{A}(x)=\frac{\delta \log J^{2}}{\delta \phi(x)} .
$$

It takes the form

$$
\mathcal{A}=4 \operatorname{tr}^{\mathrm{g}} \sum_{n} \gamma_{5}^{\alpha \beta}\left(\varphi_{n}^{\alpha} * \varphi_{n}^{\dagger}{ }^{\beta}-\varphi_{n}^{\dagger}{ }^{\alpha} * \varphi_{n}^{\beta}\right)
$$

Expression (29) is ill defined and has to be appropriately regulated. A gaugeinvariant result can be obtained by using as a basis the set of eigenfunctions of the Dirac operator $\not D$ (in the appropriate representation) and then introducing a $\not D$-based heat-kernel regularization so that one ends with a finite answer $\mathcal{A}_{\text {reg }}$

$$
\begin{aligned}
\mathcal{A}_{r e g}^{(3)}(x)= & 4 \lim _{t \rightarrow 0} \operatorname{tr}^{\mathrm{g}} \sum_{n} \gamma_{5}^{\alpha \beta}\left(\left(\exp \left(-t \gamma_{\mu} \gamma_{\nu} D_{\mu}^{a d} D_{\nu}^{a d}\right) \varphi_{n}\right)^{\alpha} * \varphi_{n}^{\dagger^{\beta}}\right. \\
& -\varphi_{n}^{\dagger^{\alpha}} *\left(\left(\exp \left(-t \gamma_{\mu} \gamma_{\nu} D_{\mu}^{a d} D_{\nu}^{a d}\right) \varphi_{n}\right)^{\beta}\right)
\end{aligned}
$$

The final expression of the anomaly depends on the space-time dimensionality $d$. Indeed, for a 2-dimensional (4-dimensional) theory only the lineal (quadratic) term in an expansion on $t$ contributes to the result. A standard (but tedious) calculation leads to,

$$
\begin{aligned}
& \mathcal{A}_{r e g}^{(3)(d=2)}(x)=\frac{e N}{2 \pi} \sqrt{\frac{N}{2}} \varepsilon_{\mu \nu}\left(F_{\mu \nu}^{0}(x-L)+F_{\mu \nu}^{0}(x+L)-2 F_{\mu \nu}^{0}(x)\right) \\
& \mathcal{A}_{r e g}^{(3)(d=4)}(x)=\frac{e^{2} N}{8 \pi^{2}}\left[F_{\mu \nu}^{a}(x+L) \tilde{F}_{\mu \nu}^{a}(x+L)-F_{\mu \nu}^{a}(x-L) \tilde{F}_{\mu \nu}^{a}(x-L)\right. \\
& \left.\quad-2\left(F_{\mu \nu}^{0}(x+L)-F_{\mu \nu}^{0}(x-L)\right) \tilde{F}_{\mu \nu}^{0}(x)\right] .
\end{aligned}
$$

with $\tilde{F}_{\mu \nu}=\frac{1}{2} \varepsilon_{\mu \nu \alpha \beta} F_{\alpha \beta}$.

An analogous calculation can be performed for the anomaly associated to currents $j_{\mu}^{5(1)}$ and $j_{\mu}^{5(2)}$. One gets

$$
\begin{aligned}
& \mathcal{A}_{r e g}^{(1)(d=2)}(x)=\frac{e N}{2 \pi} \sqrt{\frac{N}{2}} \varepsilon_{\mu \nu}\left(F_{\mu \nu}^{0}(x+L)-F_{\mu \nu}^{0}(x)\right) \\
& \mathcal{A}_{r e g}^{(1)(d=4)}(x)=\frac{e^{2} N}{8 \pi^{2}}\left[F_{\mu \nu}^{a}(x+L) \tilde{F}_{\mu \nu}^{a}(x+L)+F_{\mu \nu}^{a}(x) \tilde{F}_{\mu \nu}^{a}(x)\right. \\
& \left.\quad-2 F_{\mu \nu}^{0}(x+L) \tilde{F}_{\mu \nu}^{0}(x)\right] .
\end{aligned}
$$

One gets similar results for the case of current $j_{\mu}^{5(2)}$.

It is instructive to compare these result with those arising in the commutative case and with those corresponding to noncommutative theories defined using the Moyal product. Taking the limit $L \rightarrow 0$ in (31)-(32) we see that the anomaly 
vanishes in agreement with the trivial result one should expect in ordinary space since $j_{\mu}^{5(3)}=0$ in that case. This should be contrasted with the non-trivial answer one finds for the anomaly in the $\theta \rightarrow 0$ (i.e. commutative) limit of Moyal noncommutative theories, as a result of the well-understood IR/UV mixing taking place in this case. Concerning currents $j_{\mu}^{5(1)}$ and $j_{\mu}^{5(2)}$ the corresponding anomalies vanish in the $L \rightarrow 0$ limit for $d=2$ but give a nontrivial result for $d=4$, which coincide with the standard commutative results.

Let us end this section by briefly describing the dipole-product anomaly calculation for the case of the fundamental and anti-fundamental representations. In principle one could follow the same steps than for the adjoint representation. Nevertheless we can obtain the results directly by noticing that by renaming the field $A_{\mu}(x-L / 2)=a_{\mu}(x)$ and $A_{\mu}(x+L / 2)=a_{\mu}(x)$ for the fundamental and antifundamental representations the theories are formally identical to the ordinary commutative field theories. For the fundamental representation one obtains

$$
\left\langle\partial_{\mu} j_{\mu}^{5}\right\rangle=\mathcal{A}^{f}(x)
$$

where

$$
j_{\mu}^{5}=i\left(\gamma_{\mu} \gamma_{5}\right)^{\alpha \beta} \psi(x)^{\beta} * \bar{\psi}(x)^{\alpha}
$$

and

$$
\begin{gathered}
\mathcal{A}_{\text {reg }}^{f(d=2)}(x)=-\frac{e \sqrt{N}}{2 \sqrt{2} \pi} \varepsilon_{\mu \nu} F_{\mu \nu}^{0}(x) \\
\mathcal{A}_{\text {reg }}^{f(d=4)}(x)=\frac{e^{2}}{8 \pi^{2}} \tilde{F}_{\mu \nu}^{a}(x) F_{\mu \nu}^{a}(x) .
\end{gathered}
$$

\section{Gauge anomalies in theories with Weyl fer- mions}

In this section we shall analyze the case of Weyl fermions. As before, the results in the fundamental and antifundamental representations are trivially obtained by redefinitions of the gauge fields. We shall therefore consider the case of fermions in the adjoint representation. The action for this theory is

$$
S[\bar{\psi}, \psi, A]=\int d^{d} x \bar{\psi}(x) * D_{+} \psi(x)+S_{g}[A] .
$$

In this case, the Dirac operator $D_{+}$reads

$$
D_{+}=i \not \supset+e \gamma^{\mu} P_{+}\left[A_{\mu}, \cdot\right]_{*}
$$

where

$$
P_{ \pm}=\frac{1}{2}\left(1 \pm \gamma_{5}\right)
$$

and $S_{g}[A]$ denotes the gauge fields action. 
Notice that the chiral Dirac operator $D_{+}$is not Hermitian. In fact, its adjoint is given by

$$
D_{+}^{\dagger}=D_{-}=i \not \partial+e \gamma^{\mu} P_{-}\left[A_{\mu}, \cdot\right]_{*} .
$$

The action is invariant under the following chiral gauge transformations

$$
\begin{aligned}
\psi & \rightarrow \psi^{\prime}=e^{i P_{+}[\alpha, \cdot]} \psi=P_{+} e^{i \alpha} * \psi * e^{-i \alpha}+P_{-} \psi \\
\bar{\psi} \rightarrow \bar{\psi}^{\prime} & =\bar{\psi} e^{-i P_{-}[\cdot, \alpha]}=e^{i \alpha} * \bar{\psi} * e^{-i \alpha} P_{-}+\bar{\psi} P_{+} \\
A_{\mu} & \rightarrow A_{\mu}^{\prime}=e^{i \alpha} * A_{\mu} * e^{-i \alpha}+\frac{i}{e} e^{i \alpha} * \partial_{\mu} e^{-i \alpha}
\end{aligned}
$$

The current associated to this symmetry is classically conserved but due to the chiral nature of the transformation the conservation is anomalous. The current has two contributions, one from the matter fields and other from the gauge fields. Only the matter contribution to the current

$$
\left(j_{m}^{\mu}\right)_{a b}=\left(\bar{\psi}_{a c}^{\alpha} * \psi_{c b}^{\beta}+\psi_{a c}^{\beta} * \bar{\psi}_{c b}^{\alpha}\right)\left(\gamma^{\mu} P_{+}\right)^{\alpha \beta}
$$

is anomalous so we will concentrate on it. At the classical level it satisfies a covariant conservation law,

$$
\mathcal{D}_{\mu} j_{m}^{\mu}=\partial_{\mu} j_{m}^{\mu}-i e\left[A_{\mu}, j_{m}^{\mu}\right]=0 .
$$

We shall discuss the covariant form of the gauge anomaly adopting Fujikawa recipe 16]-15. which uses as basis for fermion fields the eigenfunctions of the Hermitian operators $D_{+} D_{-}$and $D_{-} D_{+}$. That is, one considers the eigenvalue problems

$$
\begin{gathered}
D_{-} D_{+} \varphi_{n}=\lambda_{n}^{2} \varphi_{n} \\
D_{+} D_{-} \phi_{n}=\lambda_{n}^{2} \phi_{n} \\
\phi_{n}=\frac{1}{\lambda_{n}} D_{+} \varphi_{n} \text { if } \lambda_{n} \neq 0 \text { and } D_{+} \varphi_{n}=0 \text { if } \lambda_{n}=0 \\
\varphi_{n}=\frac{1}{\lambda_{n}} D_{-} \phi_{n} \text { if } \lambda_{n} \neq 0 \text { and } D_{-} \phi_{n}=0 \text { if } \lambda_{n}=0
\end{gathered}
$$

and expands the fermion variables in the form

$$
\psi(x)=\sum c_{n} \varphi_{n}(x), \quad \bar{\psi}(x)=\sum \bar{c}_{n} \phi_{n}^{\dagger} .
$$

Again, the path-integral measure is defined as in (21)

$$
D \bar{\psi} D \psi \equiv \Pi d \bar{c}_{n} d c_{n} .
$$

Performing a chiral gauge transformation of fermion variables, we obtain for the anomaly

$$
\left\langle\left(\mathcal{D}_{\mu} j_{m}^{\mu}\right)_{a b}\right\rangle=-\frac{\delta \log (\bar{J} J)}{\delta \alpha_{b a}}=\mathcal{A}_{a b}(x)
$$


with $J$ and $\bar{J}$ being the Jacobians associated to the chiral gauge transformation of $\psi$ and $\bar{\psi}$. Since the chiral Dirac operators are non-Hermitian, those Jacobians are different and must be computed independently. After standard computation we have

$$
\mathcal{A}(x)=-2 i \operatorname{tr}\left(\sum_{n} P_{+}\left[\varphi_{n}(x), \varphi_{n}^{\dagger}(x)\right]-\sum_{n} P_{-}\left[\phi_{n}(x), \phi_{n}^{\dagger}(x)\right]\right)
$$

where the trace is taken only over the Dirac indices. This quantity is ill defined and has to be regularized. A gauge covariant regularized expression is given by

$$
\begin{aligned}
\mathcal{A}_{\text {reg }}(x)= & -2 i \lim _{t \rightarrow 0} \operatorname{tr}\left(\sum_{n} P_{+}\left[e^{-t D_{-} D_{+}} \varphi_{n}(x), \varphi_{n}^{\dagger}(x)\right]-\right. \\
& \left.\sum_{n} P_{-}\left[e^{-t D_{+} D_{-}} \phi_{n}(x), \phi_{n}^{\dagger}(x)\right]\right) .
\end{aligned}
$$

Changing the eigenstate basis to plane-wave functions and using the identity

$$
P_{+} e^{-t D_{-} D_{+}}-P_{-} e^{-t D_{+} D_{-}}=\gamma^{5} e^{-t \not D}
$$

where $\not D$ is the standard Dirac operator

$$
\not D=i \not \partial+e \gamma^{\mu}\left[A_{\mu}, \cdot\right]
$$

we obtain

$$
\mathcal{A}_{\text {reg }}(x)=-2 i \lim _{t \rightarrow 0} \operatorname{tr} \sum_{a} \int \frac{d^{d} k}{(2 \pi)^{d}}\left(\gamma^{d+1}\left[e^{-t \not D} e^{i k \cdot x} t^{a}, e^{-i k \cdot x} t^{a}\right]\right)
$$

and computing this expression for the cases of $d=2$ and $d=4$, we obtain the following chiral gauge anomalies

$$
\begin{array}{ll}
d=2 & \mathcal{A}_{\text {reg }}=i N \frac{e}{4 \pi} \varepsilon^{\mu \nu}\left(F_{\mu \nu}^{0}(x-L) t^{0}+F_{\mu \nu}^{0}(x+L) t^{0}-2 F_{\mu \nu}^{a}(x) t^{a}\right) \\
d= & 4 \quad \mathcal{A}_{r e g}=i \sqrt{2 N} \frac{e^{2}}{32 \pi^{2}}\left(\left(F_{\mu \nu}^{a}(x-L) \tilde{F}^{\mu \nu a}(x-L)-\right.\right. \\
& \left.\left.F_{\mu \nu}^{a}(x+L) \tilde{F}^{\mu \nu a}(x+L)\right) t^{0}-2\left(F_{\mu \nu}^{0}(x-L)-F_{\mu \nu}^{0}(x+L)\right) \tilde{F}^{\mu \nu a}(x) t^{a}\right)(55)
\end{array}
$$

Notice that unlike the ordinary commutative case and the noncommutative Moyal case, there is non-vanishing covariant anomaly in $d=4$ [14, 15]. Again, we would like to stress that in the $L \rightarrow 0$ limit we recover the result for the anomalies in the commutative theory because of the non-existence of the IR/UV mixing in noncommutative dipole field theories.

As we stated in the introduction, dipole theories are not only interesting in connection with string theory but also because of their possible relevance as field theories in particle physics. In this respect, it has been shown that they 
show signs of CP and CPT violations, the Lorentz symmetry breaking is soften and the possibility of constructing a dipole version of the Standard Model more attainable $[5$. In view of this, the issue of chiral and gauge anomalies in dipole theories is a relevant question, which has been discussed in this work. Using a path-integral approach, we have computed chiral and gauge anomalies in $U(N)$ dipole fermion theories. We have found non-trivial results which, in view of the connection between the anomaly and topological densities pose in turn the problem of studying topological solutions. We hope to discuss this aspect in future works.

Acknowledgements: This work partially supported by UNLP, UBA, CICBA, CONICET (PIP 4330/96), ANPCYT (PICT 03-05179), Argentina. G.S.L. and E.F.M. are partially supported by Fundación Antorchas, Argentina. F.A.S wishes to acknowledge P.Forgacs and H.Giacomini and the Laboratoire de Mathématiques et Physique Théorique de l'Université de Tours for their hospitality during part of this work.

\section{References}

[1] J. A. Harvey, arXiv:hep-th/0102076: M. R. Douglas and N. A. Nekrasov, arXiv:hep-th/0106048; R. J. Szabo, arXiv:hep-th/0109162.

[2] A. Bergman and O. J. Ganor, JHEP 0010 (2000) 018.

[3] K. Dasgupta, O. J. Ganor and G. Rajesh, and arched strings," JHEP 0104 (2001) 034.

[4] A. Bergman, K. Dasgupta, O. J. Ganor, J. L. Karczmarek and G. Rajesh, arXiv:hep-th/0103090.

[5] K. Dasgupta and M. M. Sheikh-Jabbari, arXiv:hep-th/0112064.

[6] M. M. Sheikh-Jabbari, Phys. Lett. B 455 (1999) 129.

[7] M. M. Sheikh-Jabbari, noncommutative torus," JHEP 9906 (1999) 015.

[8] D. Bigatti and L. Susskind, Phys. Rev. D 62 (2000) 066004.

[9] F. Ardalan and N. Sadooghi, Int. J. Mod. Phys. A 16, 3151 (2001).

[10] J. M. Gracia-Bondia and C. P. Martin, Phys. Lett. B 479, 321 (2000)

[11] L. Bonora, M. Schnabl and A. Tomasiello, Phys. Lett. B 485, 311 (2000)

[12] E. F. Moreno and F. A. Schaposnik, JHEP 0003, 032 (2000); Nucl. Phys. B 596, 439 (2001).

[13] C. P. Martin, J. Phys. A 34, 9037 (2001).

[14] L. Bonora and A. Sorin, Phys. Lett. B 521, 421 (2001). 
[15] C. P. Martin, Nucl. Phys. B 623, 150 (2001).

[16] K. Fujikawa, Phys. Rev. Lett. 42 (1979) 1195; Phys. Rev. D 21 (1980) 2848 [Erratum-ibid. D 22 (1980) 1499].

[17] R. E. Gamboa Saravi, M. A. Muschietti, F. A. Schaposnik and J. E. Solomin, Annals Phys. 157 (1984) 360. 